\title{
Free Edge of the Nail
}

National Cancer Institute

\section{Source}

National Cancer Institute. Free Edge of the Nail. NCI Thesaurus. Code C32633.

The side of the nail extending over the finger or toe which can be trimmed. 